\title{
Organ-Specific Tumor Response to Pembrolizumab in Advanced Urothelial Carcinoma After Platinum-Based Chemotherapy
}

This article was published in the following Dove Press journal: OncoTargets and Therapy

\author{
Nobuki Furubayashi $\mathbb{D D}^{\prime}$ \\ Takahito Negishi' \\ Naotaka Sakamoto ${ }^{2}$ \\ Hozumi Shimokawa ${ }^{3}$ \\ Futoshi Morokuma (iD) ${ }^{4}$ \\ Yoohyun Song ${ }^{5}$ \\ Yoshifumi Hori ${ }^{6}$ \\ Toshihisa Tomoda (D) $^{7}$ \\ Noriaki Tokuda ${ }^{4}$ \\ Narihito Seki ${ }^{5}$ \\ Kentaro Kuroiwa ${ }^{6}$ \\ Motonobu Nakamura' \\ 'Department of Urology, National \\ Hospital Organization Kyushu Cancer \\ Center, Fukuoka, Japan; ${ }^{2}$ Department of \\ Urology, National Hospital Organization \\ Kyushu Medical Center, Fukuoka, Japan; \\ ${ }^{3}$ Department of Medical Oncology, \\ National Hospital Organization Kyushu \\ Medical Center, Fukuoka, Japan; \\ ${ }^{4}$ Department of Urology, Saga-ken \\ Medical Centre Koseikan, Saga, Japan; \\ ${ }^{5}$ Department of Urology, Kyushu Central \\ Hospital of the Mutual Aid Association of \\ Public School Teachers, Fukuoka, Japan; \\ ${ }^{6}$ Department of Urology, Miyazaki \\ Prefectural Miyazaki Hospital, Miyazaki, \\ Japan; ${ }^{7}$ Department of Urology, Oita \\ Prefectural Hospital, Oita, Japan
}

Correspondence: Nobuki Furubayashi Department of Urology, National Hospital Organization Kyushu Cancer Center, Notame 3-I-I, Minami-Ku,

Fukuoka, 8II-1395, Japan

Tel +8I-92-54|-323I

Fax +8I-92-55I-4585

Email nobumduro@gmail.com
Background: To evaluate the organ-specific therapeutic effect of pembrolizumab after the failure of platinum-based chemotherapy for advanced urothelial carcinoma (UC).

Materials and Methods: Patients with advanced UC who received pembrolizumab after the failure of platinum-based chemotherapy and who had measurable disease were retrospectively analyzed. The objective response rate (ORR) and organ-specific response rate (OSRR) were evaluated according to Response Evaluation Criteria in Solid Tumors, version 1.1.

Results: We analyzed 69 patients (male, $\mathrm{n}=51$; median age, 71 years) with 226 metastases. The ORR was $23.2 \%$. In total, 32, 31, 16, 14, 13 and 7 patients had measurable lung (OSSR $31.3 \%$ ), lymph node (OSSR 29.0\%), local recurrence (OSSR 12.5\%), primary tumor organ (OSSR 7.1\%), liver (OSSR 23.1\%) and bone (OSSR 28.6\%) disease, respectively. The median overall survival (OS) for pembrolizumab was 10.9 months $(95 \%$ confidence interval, 5.9-13.7 months). Regarding organ-specific OS, a Log rank test significant differences in OS were confirmed between patients with and without primary tumor organ disease $(p=0.046)$ and liver metastasis $(\mathrm{p}<0.001)$.

Conclusion: Metastases and primary tumor organ disease showed different tumor responses to pembrolizumab. The most prominent tumor response was found in lung metastasis and the least response was found in primary organ sites. The mechanisms of these different responses were unclear and there does not appear to be a constant trend between tumor shrinkage and OS in tumor sites. Further studies are needed.

Keywords: urothelial carcinoma, platinum-based chemotherapy, pembrolizumab, organspecific response rate, tumor microenvironment

\section{Introduction}

Advanced urothelial carcinoma (UC) is generally considered an incurable disease. Palliative systemic chemotherapy is the standard of care and platinum-based combination chemotherapy has long been used as a first-line treatment. ${ }^{1,2}$ Platinumbased chemotherapy temporarily provides relatively good objective response rates, ${ }^{1,3}$ but the duration of the response is short. ${ }^{4}$ Furthermore, second-line treatment after the failure of platinum-based combination therapy has been of limited benefit. $^{5,6}$

In recent years, immune checkpoint inhibitors (ICIs) against programmed death 1 (PD-1) and PD-1 ligands (PD-L1 and PD-L2) have been studied as treatments for multiple malignancies, including advanced $U$ C. $^{7-9}$ At present in Japan, only 
pembrolizumab (a highly selective, humanized monoclonal IgG4 $\kappa$ isotype antibody against PD-1) has been approved for platinum-refractory advanced UC, based on the results of the KEYNOTE-045 trial. ${ }^{10,11}$ The overall survival (OS) was significantly improved in patients treated with pembrolizumab comparison to those treated with chemotherapy ( 10.3 vs 7.4 months, respectively; $p=0.002$ ), and the objective response rate (ORR) was significantly increased in patients treated with pembrolizumab in comparison to those treated with chemotherapy $(21.1 \%$ vs $11.4 \%$, respectively; $\mathrm{p}=0.001$ ). While ICIs significantly improve patient outcomes, the ORR is low, only a minority of patients show a drastic response and longterm benefits, and there are still no biomarkers that predict this clinical picture. In this trial, the PD-L1 status was also measured based on a "Combined Positive Score" (CPS), which was measured as the percentage of PD-L1-positive immune cells and tumor cells in comparison to the total number of viable tumor cells. However, the CPS was not associated with better OS, progression free survival (PFS), or ORR in the pembrolizumab arm. ${ }^{11}$

Recent studies have reported that in patients with nonsmall cell lung cancer, hepatocellular cancer and melanoma, the therapeutic effect of ICI treatment varies from organ to organ. ${ }^{12-14}$ Furthermore, metastases in different anatomical locations are also associated with the overall response and survival in melanoma patients who receive combination immunotherapy. ${ }^{14}$ However, this has not been reported in patients with UC who received ICIs.

In the present study, we retrospectively assessed the clinical outcomes of pembrolizumab in patients in whom platinum-based chemotherapy for advanced UC failed in order to clarify the organ-specific therapeutic effect of pembrolizumab, including how the location of the primary tumor and sites of metastasis influence OS and the disease control rate (DCR).

\section{Materials and Methods Patients Population}

From January 2018 to October 2020, 81 consecutive patients received pembrolizumab for advanced UC after the failure of platinum-based chemotherapy at six institutions. All patients were histopathologically diagnosed with UC and showed radiologically-confirmed disease progression after platinum-based chemotherapy. ${ }^{15}$ Only patients who had measurable disease and for whom subsequent imaging studies were available for the evaluation of the response were enrolled. Thus, 12 patients were excluded from this study. Clinical data were retrieved from the patients' medical records. Pembrolizumab was administered intravenously on day 1 at a dose of $200 \mathrm{mg}$, and the cycle was basically repeated every 21 days, and was continued until disease progression or until the occurrence of unacceptable adverse events. The present study protocol was approved by the ethics committee of each institution and complied with the 1964 Declaration of Helsinki and its later amendments.

\section{Tumor Response Evaluation}

Tumor measurements were generally performed by computed tomography before and after every four to six cycles of pembrolizumab; however, evaluations were performed as needed when the clinical symptoms worsened.

All metastases that measured $\geq 5 \mathrm{~mm}$ in the long axis (lymph node [LN] metastases $\geq 10 \mathrm{~mm}$ in the short axis) on computed tomography were defined as measurable lesions and were measured before and during pembrolizumab treatment. $^{12,16}$ The objective response rate (ORR) and disease control rate (DCR) were determined (based on Response Evaluation Criteria in Solid Tumors [RECIST], version 1.1) in up to five lesions in total and up to two lesions per organ for target lesions. Furthermore, for the organ-specific response rate (OSRR) and organ-specific disease control rate (OSDCR), the tumor burden was defined as the sum of the long axis for all non-LN metastases or the short axis of all LN metastases that were measured and evaluated according to RECIST 1.1. For each metastatic organ, the best response was classified as a complete response (CR) (disappearance or reduction to $<10 \mathrm{~mm}$ in the short axis for all LN metastases), a partial response (PR) ( $>30 \%$ reduction), stable disease (SD) (neither a $\mathrm{CR}, \mathrm{PR}$, nor progressive disease [PD]), or PD (>20\% growth). ${ }^{12,16}$

\section{Statistical Analyses}

All statistical analyses were performed using the JMP ${ }^{\circledR}$ Pro, version 15.1.0 software package (SAS Institute, Inc., Cary, NC, USA). The ORR and OSRR were defined as the proportion of patients who achieved a CR or PR with pembrolizumab based on the corresponding evaluation criteria. The DCR and OSDCR were defined as the proportion of patients who achieved a CR or PR or SD with pembrolizumab based on the corresponding evaluation criteria. OS was calculated from the day on which pembrolizumab was started until the date of the last follow-up examination or death from any 
cause and was evaluated using the Kaplan-Meier method. The Log rank test was used to determine differences in OS in the presence or absence of disease in the primary tumor organ and metastatic sites. $P$ values of $<0.05$ were considered to indicate statistical significance.

\section{Results}

\section{Patient Characteristics}

The clinical characteristics of the 69 (male, $n=51$; female, $\mathrm{n}=18$; median age, 71 years; interquartile range (IQR), 57-87 years) patients are listed in Table 1. The median follow-up period was 7.4 months. All patients received pembrolizumab for UC after the failure of platinum-based chemotherapy. According to the Eastern Cooperative Oncology Group

Table I Patient Characteristics

\begin{tabular}{|c|c|}
\hline Characteristics $(n=69)$ & \\
\hline Age (years), median (IQR) & $\begin{array}{c}71 \\
(57-87)\end{array}$ \\
\hline Male sex, no. (\%) & 51 (73.9) \\
\hline ECOG PS score, no. (\%) & \\
\hline 0 & $44(63.8)$ \\
\hline$\geq 1$ & $25(36.2)$ \\
\hline Primary tumor site, no. (\%) & \\
\hline Upper urinary tract & $26(37.7)$ \\
\hline Bladder & $24(34.8)$ \\
\hline Upper urinary tract + bladder & $19(27.5)$ \\
\hline Pure UC in histologic testing, no. (\%) & $58(84.1)$ \\
\hline $\begin{array}{l}\text { Number of prior chemotherapy regimens before } \\
\text { pembrolizumab, no. (\%) }\end{array}$ & \\
\hline I & $49(71.0)$ \\
\hline 2 & II (I5.9) \\
\hline 3 & $9(13.1)$ \\
\hline $\mathrm{Hb}<10 \mathrm{~g} / \mathrm{dl}$, no. (\%) & $24(34.8)$ \\
\hline Time from previous chemotherapy $<3$ months, no (\%) & $53(77.9)$ \\
\hline Disease site, no. (\%) & \\
\hline Lung & $32(46.4)$ \\
\hline Lymph node & $31(44.9)$ \\
\hline Local recurrence & $16(23.2)$ \\
\hline Primary tumor organ (pelvis, ureter and bladder) & $14(20.3)$ \\
\hline Liver & $13(18.8)$ \\
\hline Bone & $7(10.1)$ \\
\hline Subcutaneous & $3(4.3)$ \\
\hline Muscle & $2(2.9)$ \\
\hline Others & $3(4.3)$ \\
\hline
\end{tabular}

Abbreviations: IQR, interquartile range; ECOG PS, Eastern Cooperative Oncology Group Performance Status; UC, urothelial carcinoma; Hb, hemoglobin.
Table 2 The Objective Response Rate and Disease Control Response Rate in Patients Treated with Pembrolizumab

\begin{tabular}{|l|c|}
\hline Response (n=69) & Number of Patients (\%) \\
\hline CR & $3(4.5)$ \\
\hline PR & $13(18.8)$ \\
\hline SD & $12(17.3))$ \\
\hline PD & $41(59.4)$ \\
\hline Overall response rate (CR+PR) & $16(23.2)$ \\
\hline Disease control rate (CR+PR+SD) & $28(40.6)$ \\
\hline
\end{tabular}

Abbreviations: $C R$, complete response; $P R$, partial response; $S D$, stable disease; $\mathrm{PD}$, progressive disease.

Performance (ECOG PS), $44(63.8 \%)$ and 25 (36.2\%) patients had PS 0 and PS $\geq 1$, respectively. Twenty-six patients had upper urinary tract UC (37.7\%), 24 patients had bladder UC (34.8\%), and 19 had both types of UC (27.5\%). The number of treatments attempted before pembrolizumab was as follows: 1 treatment $(\mathrm{n}=49,71.0 \%), 2$ treatments $(\mathrm{n}=11,15.9 \%)$; and 3 treatments $(\mathrm{n}=9,13.1 \%)$. In the majority of patients, a histological examination revealed pure UC $(84.1 \%)$ and the time from the previous chemotherapy was $<3$ months $(77.9 \%)$. Before the start of treatment with pembrolizumab, metastatic target lesions were found at the following sites: lung $(46.4 \%, \mathrm{n}=32)$, lymph nodes (44.9\%, $\mathrm{n}=31)$, local recurrence $(23.2 \%, \mathrm{n}=16)$, primary tumor organ (pelvis, ureter and bladder, 20.3\%, $\mathrm{n}=14$ ), liver (18.8\%, $\mathrm{n}=13)$, bone $(10.1 \%, \mathrm{n}=7)$, subcutaneous $(4.3 \%$, $\mathrm{n}=3)$, muscle $(2.9 \%, \mathrm{n}=2)$, and others $(4.3 \%, \mathrm{n}=3)$.

\section{The ORR/DCR and OSRR/OSDCR in Patients Treated with Pembrolizumab}

The ORR and DCR in patients treated with pembrolizumab were $23.2 \%$ and $40.6 \%$, respectively (CR: $n=3, P R: n=13$, SD: $n=12$, PD: $n=41$ ) (Table 2). The OSRR and OSDCR of pembrolizumab are shown in Table 3. Among the 69 patients, the metastatic target organs before the start of treatment with pembrolizumab were the lung ( $\mathrm{n}=32$ cases; number of tumor sites: 101, median size: $13 \mathrm{~mm}$ [IQR 5-24 mm]), lymph nodes ( $\mathrm{n}=31$; number of tumor sites: 50 , median size: $18 \mathrm{~mm}$ [IQR 15-22 mm]), local recurrence $(\mathrm{n}=16$; number of tumor sites: 16, median size: $39 \mathrm{~mm}$ [IQR 21-64 mm]), primary tumor organ (pelvis, ureter and bladder) $(\mathrm{n}=14$; number of tumor sites: 14, median size: $39 \mathrm{~mm}$ [IQR 31-61 mm]), liver ( $n=13$; number of tumor sites: 26 , median size: $18 \mathrm{~mm}$ [IQR $13-33 \mathrm{~mm}])$, and bone $(\mathrm{n}=7$; number of 
Table 3 The Organ-Specific Objective Response Rate and Organ-Specific Disease Control Response Rate in Patients Treated with Pembrolizumab

\begin{tabular}{|c|c|c|c|c|c|c|}
\hline Disease Site & Lung & $\begin{array}{l}\text { Lymph } \\
\text { Node }\end{array}$ & $\begin{array}{c}\text { Local } \\
\text { Recurrence }\end{array}$ & $\begin{array}{l}\text { Primary } \\
\text { Organ }\end{array}$ & Liver & Bone \\
\hline Number of patients (\%) & $32(46.4)$ & $31(44.9)$ & $16(23.2)$ & $14(20.3)$ & $13(18.8)$ & $7(10.1)$ \\
\hline Number of tumor sites & 101 & 50 & 16 & 14 & 26 & 19 \\
\hline Median tumor size, mm (IQR) & $13(5-24)$ & $18(15-22)$ & $39(21-64)$ & $39(3 \mid-6 I)$ & $18(13-33)$ & $19(13-27)$ \\
\hline Organ-specific response rate $(C R+P R), \%$ & 31.3 & 29.0 & 12.5 & 7.1 & 23.1 & 28.6 \\
\hline $\begin{array}{l}\text { Organ-specific disease control rate }(C R+P R \\
+S D), \%\end{array}$ & 46.8 & 64.5 & 43.7 & 78.6 & 30.8 & 85.7 \\
\hline CR, n (\%) & $5(15.6)$ & $3(9.7)$ & $\mathrm{I}(6.2)$ & 0 & $2(15.4)$ & I (I4.3) \\
\hline PR, n (\%) & $5(15.6)$ & $6(19.3)$ & $\mathrm{I}(6.2)$ & I (7.2) & I (7.7) & I (I4.3) \\
\hline $\mathrm{SD}, \mathrm{n}(\%)$ & $5(15.6)$ & II (35.5) & $5(3 \mid .3)$ & 10 (7I.4) & I (7.7) & $4(57.1)$ \\
\hline PD, n (\%) & 17 (53.2) & I I (35.5) & $9(56.3)$ & $3(2 \mid .4)$ & $9(69.2)$ & I (I4.3) \\
\hline
\end{tabular}

Abbreviations: IQR, interquartile range; CR, complete response; PR, partial response; SD, stable disease; PD, progressive disease.

tumor sites: 19, median size: $19 \mathrm{~mm}$ [IQR 13-27 mm]). The OSRR for each location was as follows: lung (31.3\%), lymph nodes $(29.0 \%)$, local recurrence $(12.5 \%)$, primary tumor organ (7.1\%), liver (23.1\%) and), bone $28.6 \%$ ). The OSDCR for each location was as follows: lung (46.8\%), lymph nodes $(64.5 \%)$, local recurrence $43.7 \%)$, primary tumor organ $(78.6 \%)$, liver $(30.8 \%)$, and bone $(85.7 \%)$.

\section{The OS and Organ-Specific OS in}

\section{Patients Treated with Pembrolizumab}

The OS in patients treated with pembrolizumab is shown in Figure 1. The median OS in patients treated with pembrolizumab was 10.9 months (95\% confidence interval [CI],

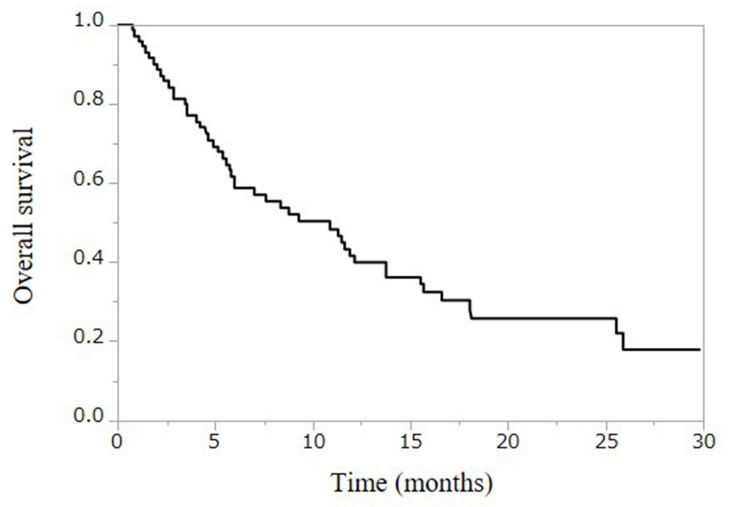

No. at risk 69

5.9-13.7 months), and the OS rate at 12 months was $41.4 \%$. Regarding organ-specific OS, a Log rank test revealed no significant difference in OS between patients with and without lung metastasis $(\mathrm{p}=0.596)$, lymph node metastasis $(\mathrm{p}=0.207$, local recurrence $(\mathrm{p}=0.280)$, or bone metastasis $(\mathrm{p}=0.616)$. However, a significant difference in OS was confirmed between patients with and without primary tumor organ disease $(\mathrm{p}=0.046)$ and liver metastasis $(\mathrm{p}<0.001)$ (Figure 2$)$.

\section{Discussion}

We retrospectively analyzed the data of 69 patients with advanced UC who received an immune checkpoint inhibitor, pembrolizumab, after the failure of platinum-based chemotherapy. To our knowledge, this is the first study to evaluate the organ-specific response to pembrolizumab in advanced UC, including how the primary location and the location of metastases influence OS. The present study found that the tumor responses to pembrolizumab differed according to the primary and metastatic sites. Specifically, we found the most prominent tumor response in lung metastases and the least response in primary organ sites, and a significant difference in OS was confirmed between patients with and without primary organ sites and liver metastasis.

Recently, it has been reported that organ-specific tumor response to ICIs differs according to the location of metastasis in some malignant tumors. For example, in non-small cell lung cancer, it was reported that treatment appears more active in lymph nodes in comparison to other organs, such as 

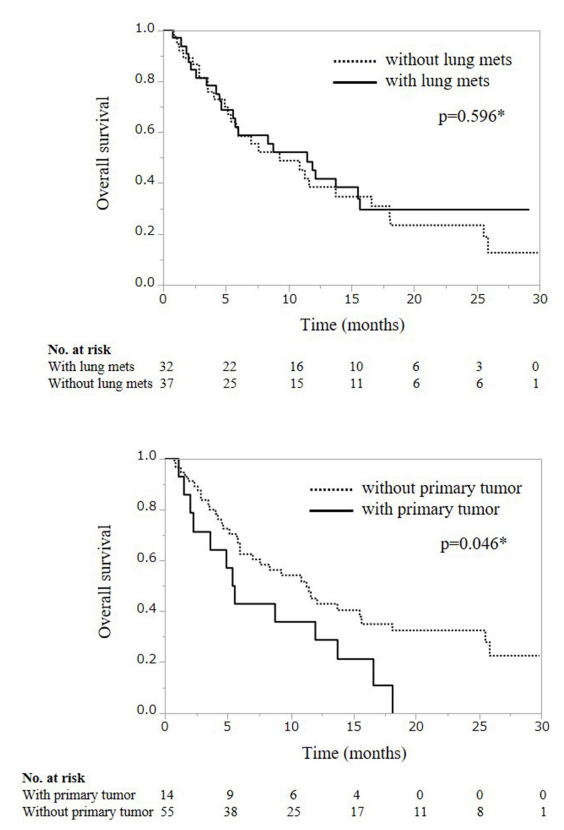
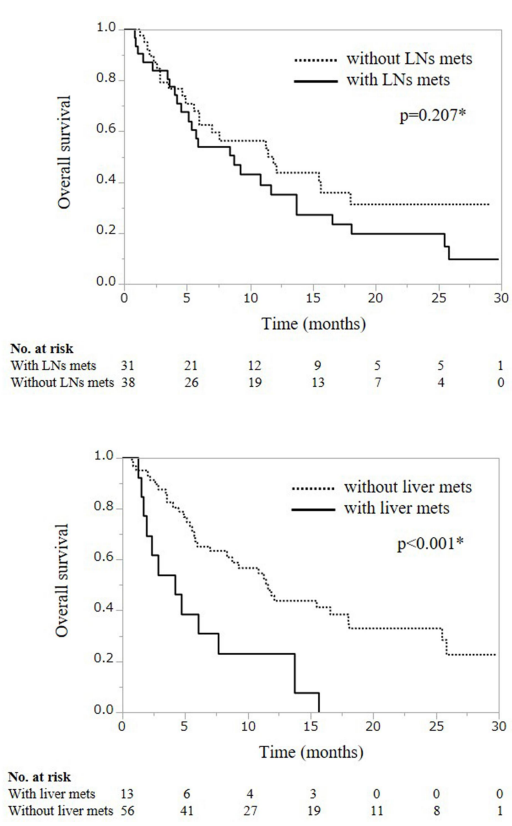
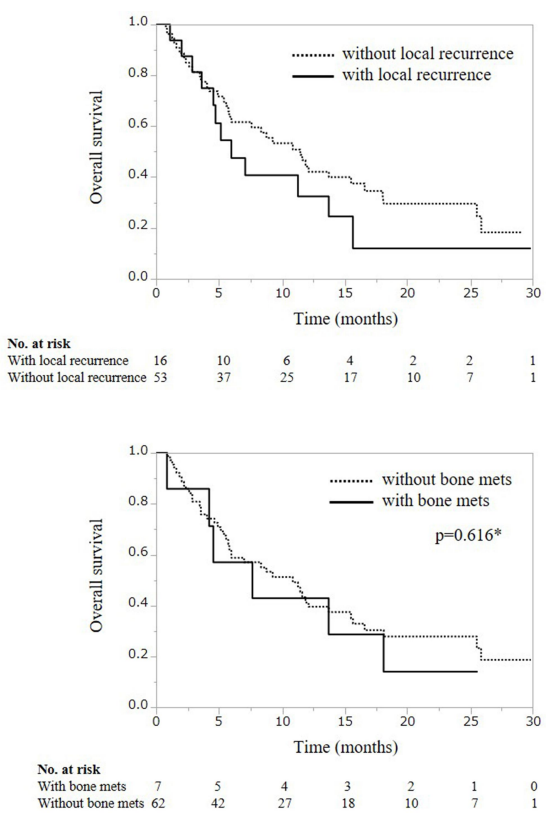

Figure 2 Organ-specific overall survival in patients treated with pembrolizumab. *P values are reported.

the liver, adrenal glands and bone. ${ }^{12}$ In hepatocellular carcinoma, it was reported that hepatic tumors of hepatocellular carcinoma may be less responsive to ICIs than extrahepatic lesions, and lung metastases responded most favorably to ICIs. ${ }^{13}$ In melanoma, it was reported that soft-tissue and lung metastases had the highest lesional response rate, whereas liver metastases had the lowest lesional response rate. ${ }^{14}$ In urothelial cancer, the organ-specific tumor response to ICI has not been reported to differ according to the site of metastasis. The present study revealed that in patients with urothelial carcinoma, a more favorable response was observed in patients with lung, lymph node, bone, and liver metastases (31.3\%, 29.0\%, 28.6\% and $23.1 \%$, respectively), while patients with primary organ disease and local recurrence showed a less favorable response $(7.1 \%$ and $12.5 \%$, respectively) with the ORR (23.2\%) taken into account. In other words, it was shown that a so-called mixed response could be confirmed in patients who received ICI treatment for urothelial cancer. There may be occasional cases involving such mixed responses to chemotherapy for advanced UC that have not been reported. We have also experienced difficulty in judging the therapeutic effect in the same course. Mixed responses have already been reported in patients with other solid tumors who received chemotherapy or targeted therapy, ${ }^{17-19}$ and these mixed reactions may be due to the heterogeneous clonality of tumor cells. ${ }^{20,21}$ For example, in melanoma, a study of 140 patients with 833 melanoma metastases revealed that there was significant interpatient and intrapatient heterogeneity in the response and progression in patients who received combination immunotherapy, which likely reflects underlying molecular heterogeneity. ${ }^{14}$ In a study that included 16 untreated colorectal cancer patients, significant levels of intermetastatic and intrametastatic heterogeneity in allelic losses, mRNA levels expression and in vitro response to chemotherapy have been demonstrated by testing the heterogeneity between several samples of the same metastatic liver lesion or between multiple metastatic liver lesions in a single patient. ${ }^{20}$ Therefore, it could be considered that in addition to the heterogeneous tumor colonies that are seen in different organs, there may be heterogeneous tumor colonies within the same organ; this remains true in urothelial carcinoma.

In recent studies, conventional chemotherapy was shown to have direct cytotoxic effects on tumor cells, but to also promote antitumoral immune responses. ${ }^{22}$ Chemotherapy can enhance the antigen presenting ability of human B cells. ${ }^{23}$ Furthermore, chemotherapy may induce direct immunoregulatory properties by promoting dendritic cell (DC) maturation and by enhancing $\mathrm{T}$ cell activation by DCs. ${ }^{24,25}$ Chemotherapy can also potentiate antigen-specific $\mathrm{T}$ cell responses, by diminishing the influence of immunosuppressive cells, such as regulatory $\mathrm{T}$ cells. ${ }^{22}$ On the other hand, ICIs induce antitumor effects by reactivating exhausted $\mathrm{T}$ cells and activating antitumor immunity. Therefore, differences in the tumor microenvironment of different organs may affect the therapeutic effect of ICIs. It is theoretically reasonable that the results of 
different therapeutic effects with ICIs were obtained for each organ and that mixed responses would be even more pronounced in patients treated with ICIs than in those treated with chemotherapy. Conventional criteria, such as RECIST, were developed based on the data from clinical trials of cytotoxic chemotherapy agents for advanced malignancies. ${ }^{26}$ However, in the era of ICI treatment, it is unclear how much the change in the tumor burden affects OS. The present study also compared OS between groups with and without disease at the primary tumor sites and metastasis at different organs. The present target organs were the lung, lymph nodes, local recurrence, primary organ sites (pelvis, ureter and bladder), liver and bone, and a Log rank test revealed a significant difference in OS between patients with and without primary organ disease and liver metastasis $(\mathrm{p}=0.046$ and $\mathrm{p}<0.001$, respectively). Interestingly, however, liver metastasis was associated with a more favorable response $(23.1 \%)$, while primary organs disease was associated with a less favorable response $(7.1 \%)$, in terms of ORR. The assessment of the change in the tumor burden is an important feature in the clinical evaluation of cancer therapeutics. However, RECIST 1.1 does not take into account potentially different treatment activity across different organ sites; thus, conventional measures may not fully characterize the impact of ICIs. For example, the study of patients with advanced renal cell carcinoma who received nivolumab plus ipilimumab showed that a tumor burden reduction of $>50 \%$ may be a useful indicator of the potential for long-term survival in patients treated with nivolumab and ipilimumab, while patients with advanced renal cell carcinoma who received nivolumab plus ipilimumab and who had a 50-75\% reduction in tumor burden had a similar overall survival to those with a $>75 \%$ reduction in the randomized, open-label, Phase 3 CheckMate 214 trial. $^{27}$ It was also reported that studies focusing on other cancers, such as melanoma, have shown similar findings, supporting the hypothesis that a durable benefit is not confined to patients with a RECIST-defined complete response. $^{28}$ These previous results indicate that responses according to the RECIST version 1.1 guidelines might not capture the spectrum of clinical outcomes, and may also support the results of the present study.

According to prognostic factors in patients with advanced transitional cell carcinoma of the urothelial tract who experienced treatment failure with platinum-containing regimens, 4 adverse risk factors (PS, hemoglobin level, liver metastasis and time from prior chemotherapy) have been reported, ${ }^{29,30}$ and these 4 risk factors were stratified, even in the phase 3 KEYNOTE-045 trial, in which pembrolizumab was associated with significantly longer overall survival and a lower rate of treatment-related adverse events in comparison to chemotherapy as a second-line therapy for platinum-refractory advanced UC. ${ }^{11}$ In this trial, according to the relationship between the metastatic organs and OS, the presence or absence of visceral (including liver, lung, bone, and any non-lymph node or soft tissue) metastasis, lymph node metastasis, bone metastasis, and liver metastasis were also analyzed, and it was reported that only liver metastasis was an independent prognostic factor. ${ }^{29}$ The liver is reported to have an immunologically unique microenvironment and to have developed a variety of mechanisms for inducing and maintaining immunological tolerance because it is constantly exposed to various antigens, such as microbial products from intestinal bacteria. ${ }^{31,32}$ In patients with advanced hepatocellular carcinoma, it was reported that the corresponding OSSR for hepatic tumors and lung, lymph node, and other intra-abdominal metastases were $22.4 \%$, $41.2 \%, 26.3 \%$, and $38.9 \%$, respectively, and the hepatic tumors of HCC may be less responsive to ICIs than extrahepatic lesions. ${ }^{13}$ In patients with non-small cell lung cancer, it was reported that the OSRR and OSDCR were $28 \%$ and $90 \%$, respectively, in lymph node metastasis, $8 \%$ and 54 in liver metastasis, and $9 \%$ and $55 \%$ in lung metastasis, the cumulative incidence probability of organ-specific progression at 6 months was $14 \%$ in lymph node metastasis, $42 \%$ in liver metastasis, $36 \%$ in lung metastasis, $26 \%$ in the primary tumor, $29 \%$ in soft tissue metastasis, and 33\% in adrenal metastasis, and the treatment in lymph nodes appears to be more active in comparison to other organ sites such as liver, adrenal gland and bone metastasis. ${ }^{12}$ In patients with melanoma, it was reported that soft-tissue and lung metastases had the highest lesional response rate $(79 \%$ and $77 \%$, respectively), whereas liver metastases had the lowest (46\%). In a multivariate analysis, patients with lung metastases had a superior ORR (odds ratio $[\mathrm{OR}], 2.75 ; \mathrm{P}=0.02$ ) and progression-free survival (hazard ratio [HR], 0.46; $\mathrm{P}=0.02$ ), whereas those with liver metastasis had an inferior $\mathrm{ORR}(\mathrm{OR}, 0.33 ; \mathrm{P}=0.02)$, progression-free survival (HR, 4.03; $\mathrm{P}<0.01$ ), and overall survival (HR, 3.17; $\mathrm{P}=0.01){ }^{14}$ In the present study, the OSRR of patients with liver metastasis was not so bad (23.1\%) in comparison to other organs (lung [31.3\%], lymph nodes [29.0\%], local recurrence [12.5\%], primary tumor organ [7.1\%] and bone [28.6\%]). However, a significant difference in OS was confirmed between patients with and without liver metastasis $(\mathrm{p}<0.001)$, these results may be due in part to the liver having an immunologically unique microenvironment.

The present study was associated with some limitations. This study was retrospective in nature. Due to the relatively small sample size, a multivariate analysis was not performed. 
The absence of statistically significant difference in organs other than the primary tumor organ and liver may be due to the sample size; thus, our findings should be validated in large and prospective studies. The patients in our study were heterogeneous in terms of the regimens and lines of prior systemic chemotherapy, dosing schedule, and the frequency of tumor assessment. The immunological mechanisms of organ-specific differential responses to pembrolizumab were not explored because it is impossible to obtain tumor samples from multiple organs in the same person at the same time.

\section{Conclusion}

The present study found that the tumor responses in patients treated with pembrolizumab were different and that they depended on the primary and metastatic sites. Specifically, we found the most prominent tumor response in lung metastases and the least response in primary organ sites, and that significant difference in OS was confirmed with and without primary organ disease and liver metastasis. The mechanisms of these different responses were unclear and there does not appear to be a constant trend between tumor shrinkage and OS in tumor sites. Further studies are needed.

\section{Abbreviations}

UC, urothelial carcinoma; ICIs, immune checkpoint inhibitors; ORR, objective response rate; DCR, disease control rate; OSRR, organ-specific response rate; OS, overall survival; ICIs, immune checkpoint inhibitors; PD-1, programmed death 1; CPS, combined positive score; PFS, progression free survival; LN, lymph node; OSDCR, organ-specific disease control rate; ECOG PS, Eastern Cooperative Oncology Group Performance Status; $\mathrm{Hb}$, hemoglobin; IQR, interquartile range.

\section{Data Sharing Statement}

The datasets used and/or analyzed during the current study are available from the corresponding author on reasonable request.

\section{Ethics Approval and Consent to Participate}

The present study was approved by the Institutional Review Board of National Hospital Organization Kyushu Cancer Center (2020-28), and written informed consent was obtained from all patients.

\section{Author Contributions}

All authors made a significant contribution to the work reported, whether that is in the conception, study design, execution, acquisition of data, analysis and interpretation, or in all these areas; took part in drafting, revising or critically reviewing the article; gave final approval of the version to be published; have agreed on the journal to which the article has been submitted; and agree to be accountable for all aspects of the work.

\section{Funding}

No funding was received.

\section{Disclosure}

The authors declare that they have no conflict of interest for this study.

\section{References}

1. Maase HVD, Sengelov L, Roberts JT, et al. Long-term survival results of a randomized trial comparing gemcitabine plus cisplatin, with methotrexate, vinblastine, doxorubicin, plus cisplatin in patients with bladder cancer. $J$ Clin Oncol. 2005;23(21):4602-4608. doi:10.1200/JCO.2005.07.757

2. National Comprehensive Cancer Network. Guidelines on bladder cancer. July 16, 2020. Available from: https://www.nccn.org/profes sionals/physician_gls/pdf/bladder.pdf. Accessed November 1, 2020.

3. Sternberg CN, Yagoda A, Scher HI, et al. M-VAC: methotrexate (MTX), vinblastine (VLB), adriamycin (ADM), and cisplatin (DDP) for metastatic and node positive carcinoma of the urothelium. Prog Clin Biol Res. 1988;260:481-485.

4. Plimack ER, Bellmunt J, Gupta S, et al. Safety and activity of pembrolizumab in patients with locally advanced or metastatic urothelial cancer (KEYNOTE-012): a non-randomised, open-label, phase 1b study. Lancet Oncol. 2017;18(2):212-220. doi:10.1016/ S1470-2045(17)30007-4

5. Santis MD, Bellmunt J, Mead G, et al. Randomized phase II/III trial assessing gemcitabine/carboplatin and methotrexate/carboplatin/vinblastine in patients with advanced urothelial cancer who are unfit for cisplatin-based chemotherapy: EORTC study 30986. J Clin Oncol. 2012;30(2):191-199. doi:10.1200/JCO.2011.37.3571

6. Sweeney CJ, Roth BJ, Kabbinavar FF, et al. Phase II study of pemetrexed for second-line treatment of transitional cell cancer of the urothelium. J Clin Oncol. 2006;24(21):3451-3457. doi:10.1200/ JCO.2005.03.6699

7. Seidel JA, Otsuka A, Kabashima K. Anti-PD-1 and anti-CTLA-4 therapies in cancer: mechanisms of action, efficacy, and limitations. Front Oncol. 2018;8:86. doi:10.3389/fonc.2018.00086

8. Califano G, Ouzaid I, Verze P, et al. Immune checkpoint inhibition in upper tract urothelial carcinoma. World J Urol. 2020. doi:10.1007/ s00345-020-03502-7

9. Califano G, Ouzaid I, Verze P, et al. New immunotherapy treatments in non-muscle invasive bladder cancer. Arch Esp Urol. 2020;73 (10):945-953.

10. Yuasa T, Urakami S, Yonese J. Recent advances in medical therapy for metastatic urothelial cancer. Int J Clin Oncol. 2018;23 (4):599-607. doi:10.1007/s10147-018-1260-0 
11. Bellmunt J, Wit RD, Vaughn DJ, et al. Pembrolizumab as second-line therapy for advanced urothelial carcinoma. $N$ Engl J Med. 2017;376 (11):1015-1026. doi:10.1056/NEJMoa1613683

12. Schmid S, Diem S, Li O, et al. Organ-specific response to nivolumab in patients with non-small cell lung cancer (NSCLC). Cancer Immunol Immunother. 2018;67(12):1825-1832. doi:10.1007/s00262-018-2239-4

13. Lu LC, Hsu C, Shao YY, et al. Differential organ-specific tumor response to immune checkpoint inhibitors in hepatocellular carcinoma. Liver Cancer. 2019;8(6):480-490. doi:10.1159/000501275

14. Silva PDI, Lo S, Quek C, et al. Site-specific response patterns, pseudoprogression, and acquired resistance in patients with melanoma treated with ipilimumab combined with anti-PD-1 therapy. Cancer. 2020;126(1):86-97. doi:10.1002/cncr.32522

15. Moch H, Cubilla AL, Humphrey PA, et al. The 2016 WHO classification of tumours of the urinary system and male genital organs-part A: renal, penile, and testicular tumours. Eur Urol. 2016;70 (1):93-105. doi:10.1016/j.eururo.2016.02.029

16. Furubayashi N, Negishi T, Miura A, et al. Organ-specific therapeutic effect of paclitaxel and carboplatin chemotherapy after platinum-based chemotherapy and pembrolizumab for metastatic urothelial carcinoma. Res Rep Urol. 2020;12:455-461. doi:10.2147/ RRU.S270495

17. Kessel CSV, Samim M, Koopman M, et al. Radiological heterogeneity in response to chemotherapy is associated with poor survival in patients with colorectal liver metastases. Eur J Cancer. 2013;49 (11):2486-2493. doi:10.1016/j.ejca.2013.03.027

18. Chen ZY, Zhong WZ, Zhang XC, et al. EGFR mutation heterogeneity and the mixed response to EGFR tyrosine kinase inhibitors of lung adenocarcinomas. Oncologist. 2012;17(7):978-985. doi:10.1634/ theoncologist.2011-0385

19. Mete UK, Singh DV, Kakkar N, et al. Sustained systemic response paralleled with ovarian metastasis progression by sunitinib in metastatic renal cell carcinoma: is this an anti-angiogenic potentiation of cancer? Urol Ann. 2015;7(3):387-390. doi:10.4103/0974-7796.153669

20. Goasguen N, Chaisemartin CD, Brouquet A, et al. Evidence of heterogeneity within colorectal liver metastases for allelic losses, mRNA level expression and in vitro response to chemotherapeutic agents. Int J Cancer. 2010;127(5):1028-1037. doi:10.1002/ijc.25114

21. Schmid K, Oehl N, Wrba F, et al. EGFR/KRAS/BRAF mutations in primary lung adenocarcinomas and corresponding locoregional lymph node metastases. Clin Cancer Res. 2009;15(14):4554-4560. doi:10.1158/1078-0432.CCR-09-0089

22. Krantz D, Hartana CA, Winerdal ME, et al. Neoadjuvant chemotherapy reinforces antitumour $\mathrm{T}$ cell response in urothelial urinary bladder cancer. Eur Urol. 2018;74(6):688-692. doi:10.1016/j. eururo.2018.06.048
23. Zirakzadeh AA, Kinn J, Krantz D, et al. Doxorubicin enhances the capacity of B cells to activate $\mathrm{T}$ cells in urothelial urinary bladder cancer. Clin Immunol. 2017;176:63-70. doi:10.1016/j. clim.2016.12.003

24. Hu J, Kinn J, Zirakzadeh AA, et al. The effects of chemotherapeutic drugs on human monocyte-derived dendritic cell differentiation and antigen presentation. Clin Exp Immunol. 2013;172(3):490-499. doi:10.1111/cei.12060

25. Grisay G, Pierrard J, Confente C, et al. Future strategies involving immune checkpoint inhibitors in advanced urothelial carcinoma. Curr Treat Options Oncol. 2020;22(1):7. doi:10.1007/s11864-020-00799-9

26. Therasse P, Arbuck SG, Eisenhauer EA, et al. New guidelines to evaluate the response to treatment in solid tumors. European Organization for Research and Treatment of Cancer, National Cancer Institute of the United States, National Cancer Institute of Canada. J Natl Cancer Inst. 2000;92(3):205-216. doi:10.1093/jnci/ 92.3.205

27. Grunwald V, Choueiri TK, Rini BI, et al. Association between depth of response and overall survival: exploratory analysis in patients with previously untreated advanced renal cell carcinoma (aRCC) in CheckMate 214. Ann Oncol. 2019;30(suppl 5):v356-402 (abstr). doi:10.1093/annonc/mdz249.046

28. Osgood C, Mulkey F, Mishra-Kalyani PS, et al. FDA analysis of depth of response (DpR) and survival across 10 randomized controlled trials in patients with previously untreated unresectable or metastatic melanoma (UMM) by therapy type. Proc Am Soc Clin Oncol. 2019;37(suppl 15):9508 (abstr 9508).

29. Bellmunt J, Choueiri TK, Fougeray R, et al. Prognostic factors in patients with advanced transitional cell carcinoma of the urothelial tract experiencing treatment failure with platinum-containing regimens. Clin Oncol. 2010;28(11):1850-1855. doi:10.1200/ JCO.2009.25.4599

30. Sonpavde G, Pond GR, Fougeray R, et al. Time from prior chemotherapy enhances prognostic risk grouping in the second-line setting of advanced urothelial carcinoma: a retrospective analysis of pooled, prospective Phase 2 trials. Eur Urol. 2013;63(4):717-723. doi:10.1016/j.eururo.2012.11.042

31. Chan T, Wiltrout RH, Weiss JM. Immunotherapeutic modulation of the suppressive liver and tumor microenvironments. Int Immunopharmacol. 2011;11(7):879-889. doi:10.1016/j.intimp.2010.12.024

32. Tiegs G, Lohse AW. Immune tolerance: what is unique about the liver. J Autoimmun. 2010;34(1):1-6. doi:10.1016/j.jaut.2009.08.008
OncoTargets and Therapy

\section{Publish your work in this journal}

OncoTargets and Therapy is an international, peer-reviewed, open access journal focusing on the pathological basis of all cancers, potential targets for therapy and treatment protocols employed to improve the management of cancer patients. The journal also focuses on the impact of management programs and new therapeutic agents and protocols on patient perspectives such as quality of life, adherence and satisfaction. The manuscript management system is completely online and includes a very quick and fair peer-review system, which is all easy to use. Visit http://www.dovepress.com/ testimonials.php to read real quotes from published authors. 